\title{
La movilización de objetos culturales en la práctica de construcción de casas de una comunidad indígena Embera Chamí: posibilidades para pensar el (Por) venir de la Educación [Matemática] Indígena'
}

\author{
The mobilization of cultural objects in the practice of building \\ houses an indigenous Embera Chami: possibilities to think about \\ the (Por) come from the Education [Mathematics] Indigenous
}

A mobilização de bens culturais na prática da construção de casas de Embera Chami indígena: possibilidades para pensar sobre o (Por) vêm da Educação [Matemática] Indígena

Recibido: mayo 2013

Aceptado: agosto 2013
Carolina Higuita Ramírez ${ }^{2}$ Abelardo Tascón Vélez ${ }^{3}$

Diana Victoria Jaramillo Quiceno ${ }^{4}$

\begin{abstract}
Resumen
Esta investigación se sitúa en una perspectiva sociocultural de la educación matemática, específicamente en la Etnomatemática y la Educación Indígena. La pregunta de investigación es ¿Cómo se movilizan objetos culturales en la práctica de construcción de casas de una comunidad indígena Embera Chamí para pensar el (por)venir de la Educación [Matemática] Indígena? Así, los objetivos del estudio son: primero, analizar la movilización de objetos culturales en la práctica de construcción de casas de una comunidad indígena Embera Chamí; y segundo, problematizar esa movilización de objetos culturales para pensar el (por)venir de la Educación [Matemática] Indígena. Son fuentes de inspiración los planteamientos de la comunidad indígena y de autores como D’Ambrosio (2008; 2011), Miguel (2008; 2010), Walsh $(2005 ; 2007)$, entre otros.

Palabras clave: Educación Matemática y otras disciplinas; Investigación e Innovación en Educación Matemática; Marcos Teóricos; Teorías de Aprendizaje;SituadoSociocultural;Alumno;Diversidad;Cultura-Religión.
\end{abstract}

\begin{abstract}
This research is situated in a sociocultural perspective of mathematics education, specifically in the Ethnomathematics and Indigenous Education. The research question is how cultural objects are mobilized in the practice
\end{abstract}

1 Artículo de investigación.

2 Universidad de Antioquia. Medellin, Colombia. Integrante Grupo “Matemática, Educación y Sociedad”. Contacto: cahira0605@ gmail.com

3 Centro Educativo Rural Indigenista La María Diana Victoria Jaramillo Quiceno. Colombia. Contacto: atableardo1@gmail.com

4 Universidad de Antioquia. Medellin, Colombia. Integrante Grupo “Matemática, Educación y Sociedad”. Contacto: diana_jaramillo@ hotmail.com 
of building houses Embera Chami indigenous community to think the (by) coming of Education [Mathematics] Indian? Thus, the objectives of the study are: first, to analyze the mobilization of cultural objects in the practice of building houses Embera Chami indigenous community, and second, to problematize the mobilization of cultural objects to think the (by) coming of Education [Mathematics] Indian. They are inspirations approaches indigenous community of authors like D’Ambrosio (2008, 2011), Miguel (2008, 2010), Walsh $(2005,2007)$, among others.

Keywords: Mathematics Education and other disciplines, Research and Innovation in Mathematics Education, Theoretical Frameworks, Theories of Learning, Situated Sociocultural, Student, Diversity, Culture-Religion.

\section{Resumo}

Esta pesquisa situa-se em uma perspectiva sociocultural da educação matemática, especificamente nas Etnomatemática e Educação Indígena. A questão de pesquisa é como os objetos culturais são mobilizados na prática da construção de casas Embera Chami comunidade indígena para que o (by) vinda de Educação [Matemática] indiano? Assim, os objetivos do estudo são: em primeiro lugar, analisar a mobilização de bens culturais na prática da construção de casas Embera Chami comunidade indígena e, segundo, para problematizar a mobilização de bens culturais para que o (by) vinda de Educação [matemática] indiano. Eles são inspirações aproxima comunidade indígena de autores como D’Ambrosio $(2008,2011)$, Miguel (2008, 2010), Walsh (2005, 2007), entre outros.

Palavras-chave: Educação Matemática e outras disciplinas, Investigação e Inovação em Educação Matemática, quadros teóricos, teorias da aprendizagem, situada Sociocultural, Estudante, diversidade, cultura, religião. Introducción

\section{El problema vislumbrado}

El pensar sobre la educación indígena en nuestro país y particularmente sobre la enseñanza y el aprendizaje de las matemáticas en escenarios indígenas se convirtió para nosotros en una preocupación desde que tuvimos la posibilidad de participar en diferentes proyectos de investigación liderados por el grupo Matemáticas, Educación y Sociedad-MES, de la Facultad de Educación de la Universidad de Antioquia. Investigaciones que han intentado transgredir paradigmas en el campo teórico y metodológico, traspasando fronteras para hacer investigación con y para las comunidades indígenas de Antioquia. Estas investigaciones intentaban (re)conocer, valorar y legitimar otras formas de saber/hacer matemático. Los proyectos a los que nos referimos y en los que hemos participado son: Higuita (2011); Jaramillo, Tamayo \& Higuita (2013) y Tamayo (2012).

Es desde la discusiones, allí tejidas, y desde las conversaciones con maestros, niños y líderes indígenas Dule y Embera Chamí que comenzamos a entender la necesidad de estudiar las prácticas que caracterizan cada una de las comunidades indígenas en relación con lo matemático. Inicialmente, en esos proyectos de investigación (antes mencionados) presentados en el marco de la Etnomatemática, se trató de estudiar una práctica social para mirar conceptos matemáticos de acuerdo a nuestra propia concepción que como investigadores tenemos de la disciplina (de la matemática occidental). Nos referimos específicamente a los trabajos de Berrío (2009) y de Higuita (2011). En este marco, es posible 
afirmar que a nivel nacional e internacional estas investigaciones vienen tomando auge, particularmente en la línea de Etnomatemática. Es por esto, que se hizo necesario a partir de las reflexiones que se generaron, el estudio de algunas investigaciones que nos posibilitaran ampliar el horizonte conceptual y metodológico sobre dicha relación.

En Gerdes (2010) --estudio realizado con el pueblo indígena Bora, en Perú-- se presenta una interpretación de los tejidos y la decoración de dos tipos de cestos desde la mirada de la geometría en la concepción de la cultura occidental. En Covián (2005) se buscó reflexionar sobre el papel del conocimiento matemático en las prácticas de la cultura maya, particularmente desde la construcción de la vivienda tradicional teniendo como perspectiva teórica en educación matemática la socio-epistemología. En efecto estas lecturas de las prácticas sociales de las comunidades indígenas en relación al conocimiento matemático nos seguían causando insatisfacción, sentíamos que sobre una práctica impregnada de aspectos relacionados con la cosmovisión y cosmogonía de los pueblos el conocimiento tenía que ser pensado desde otras miradas.

Es así, como en la inquietante necesidad de continuar buscando otras interpretaciones sobre dicha relación, encontramos, en Mendes (2001), otra posibilidad de comprender las prácticas sociales y el conocimiento matemático que en ellas se presentan. Esta propuesta tenía que ver con comprender las matemáticas en el contexto en que se desarrollaban, en la comunidad indígena del Xingu en Brasil. En esa investigación se reconoce cómo una práctica social, la práctica de numeramiento-letramiento, tiene unos significados otros, distintos a los establecidos en la práctica dominante en la escuela, y es a partir de este reconocimiento que se plantea como una necesidad tejer diálogos entre ellos. Lo anterior se presenta como una mirada diferente a la que veníamos aproximándonos en otros trabajos con las comunidades indígenas.
En Tamayo (2012) emergen otros elementos que apuntan a profundizar la discusión entre las prácticas sociales y el conocimiento matemático. El objeto de estudio de esa investigación fue: el currículo escolar indígena relativo al conocimiento [matemático] en la comunidad indígena Dule de Alto Caimán. La apuesta de esa investigadora estaba colocada en la necesidad de tejer una dialogía entre las prácticas sociales y el conocimiento [matemático]. Tamayo (2012) se refiere al conocimiento [matemático], esta última palabra en corchetes para expresar que al interior de la comunidad Dule la palabra matemática no tiene ningún sentido. Para la comunidad indígena Dule, de acuerdo con Tamayo (2012), el conocimiento no está disciplinarizado, no está fragmentado; el conocimiento todo se constituye en unidad. Esta reflexión, sin duda alguna trasgrede o trasciende paradigmas que hoy están influenciados, en la cultura occidental, por la racionalidad de la modernidad y la disciplinarización del conocimiento.

La reflexión sobre la indisciplinarización del conocimiento en las comunidades indígenas, será, sin duda, un planteamiento sobre el cual el proyecto ${ }^{5}$ que proponemos está profundizando y analizando, con la idea de aportar miradas otras, escenarios otros en particular para la Educación Indígena. Es bajo este marco que proponemos como pregunta de investigación: ¿Cómo se movilizan objetos culturales en la práctica ${ }^{6}$ de construcción de casas de una comunidad indígena Embera Chamí para pensar la Educación [Matemática] Indígena?

\section{Horizonte conceptual}

Este estudio se enmarca en una perspectiva sociocultural de la educación matemática. En este sentido, la investigación que desarrollamos intenta tejer diálogos con los planteamientos de D'Ambrosio (2008; 2011), Miguel (2008; 2010), Walsh (2005; 2007) en el campo de la Etnomatemática desde constructos

5 Consideramos importante resaltar que el proyecto surge también de las interacciones que como profesoras y estudiantes hemos tejidos en el marco de la Licenciatura en Pedagogía de la Madre Tierra de la Universidad de Antioquia.

6 La opción de referirnos a la práctica de la construcción de casas y no a otra tiene que ver con que dicha práctica refleja para el pueblo Embera su pensamiento, esto es su cosmovisión, cosmogonía y espiritualidad. Esta práctica da cuenta de su identidad como indígenas. 
tales como práctica cultural, objetos culturales, movilización y educación intercultural. A continuación presentamos, desde nuestra perspectiva, dos campos que se constituyen en elementos claves para la investigación.

Los objetos culturales en la Educación matemática: una apuesta epistemológica, histórica y política. En el marco de las actividades humanas, comprendidas de acuerdo con Miguel (2010) como formas de organización e interacción social se movilizan ${ }^{7}$ unas prácticas y por tanto unos objetos culturales que responden a las necesidades propias de las comunidades, unas necesidades de sobrevivencia y trascendencia en el sentido de D’Ambrosio (2008). Algunas de estas prácticas y objetos culturales terminan por diferentes razones demarcados y valorados por algunas comunidades, como por ejemplo las comunidades científico-académicas. Otros objetos culturales son movilizados en prácticas indisciplinarizadas que también son valoradas en las comunidades en que estos surgen. En este sentido, es posible afirmar, en coherencia con lo propuesto por Miguel (2008), que los objetos culturales en la perspectiva que asumimos son "(...) interpretados de diferentes maneras, dependiendo de quién lo moviliza, de la forma cómo lo moviliza, de la intención con que lo moviliza y del instrumento mediador de la movilización (...)". (p.46). Lo anterior reconoce dos características que son fundamentales de los objetos culturales, están son: los objetos culturales son dependientes de los sujetos y vienen siendo (es decir, están siempre en constitución). Es desde esta postura epistemológica que el proyecto de investigación se está desarrollando.

Reflexiones sobre el (Por)venir de la Educación [Matemática] Indígena. Pensar sobre la educación indígena en Antioquia y, en particular, en la comunidad Embera Chamí está relacionada con la idea de (por)venir en el sentido de la Larrosa (2001). El (por)venir asumido como aquello que no se puede predecir, que alberga la continuidad y la discontinuidad y problematiza la relación entre el pasado, el presente y el futuro. Es por esto que, en el marco de un análisis de la movilización de los objetos culturales en la práctica de construcción de casas, se propone problematizar esa movilización de objetos culturales para pensar el (por)venir de la Educación [Matemática] Indígena. El verbo problematizar es comprendido aquí como poner en cuestión, presentar una alternativa, entre otras, que posibilite hacer visible la interculturalidad como un proyecto político-epistemológico. Proyecto que consiste básicamente en crear las condiciones reales y materiales para poner en diálogo diferentes epistemologías. Aquí, fundamentalmente la(s) epistemología(s) de la cultura indígena Embera Chamí y la(s) epistemología(s) de la cultura occidental. Esta necesidad de pensar la interculturalidad surge de los intereses planteados por la comunidad en diferentes escenarios académicos y comunitarios.

Una propuesta que visualizamos, como una opción para empezar a tejer este diálogo entre epistemologías, está inspirada en los planteamiento de Miguel (2008; 2010), Lanner, Miguel \& Vilela (2012) en relación a una apuesta por una indisciplinarización. Ella consiste en estudiar (sea al interior de la escuela $\mathrm{u}$ en otros espacios educativos) las diferentes formas de vida de los objetos culturales que se movilizan en la práctica de construcción de casas en diferentes actividades humanas. Dicha propuesta se fundamenta, como lo diría Schafer (2008), en un respeto por lo que cada cultura produce.

\section{Horizonte metodológico}

La investigación se enmarca en un paradigma indígena poscolonial de corte cualitativo que: (1) reconoce la sensibilidad del contexto, (2) reconoce la importancia de la tradición oral. (3) valora y legitima la voz de la comunidad desde el diálogo y la construcción colectiva de propuestas. (4) tiene en cuenta las relaciones entre el pasado, el presente y el futuro. (5) Pone en diálogo teorías surgidas desde la comunidad indígena y desde la cultura occidental para comprender y transformar la realidad (Chilisa, 2012). En este sentido, consideramos que la investigación tiene un enfoque crítico-dialéctico, como es planteado por Sánchez (1998). La forma de aproximarnos a la movilización de objetos culturales para pensar el (por)venir de la educación [matemática]

7 La movilización de objetos culturales se comprende como un proceso en el que los sujetos participan activamente de acuerdo a unas necesidades de sobrevivencia y de trascendencia en un tiempo y en un espacio particulares. En esa movilización el lenguaje se torna clave. 
indígena se fundamenta en la utilización de algunos métodos como la historia oral (Joutard, 1999) y la investigación participante (Chilisa, 2012). Esta forma de producir conocimiento en la investigación se está realizando considerando fundamentalmente los siguientes registros: la entre-vista, los círculos de conversación, las narrativas, documentos públicos, dibujos y fotografías, entre otros. El análisis de los datos producidos se viene realizando de manera abierta y flexible, lo que implica una interpretación y una significación de los mismos en relación con los diálogos tejidos con autores y con nuestras posturas como investigadores y participantes.

\section{Referencias}

Berrío, K. (2009). La medida en el contexto de escuela indígena. El caso de una comunidad Tule y el caso de la comunidad Embera Chamí (Trabajo de Grado). Universidad de Antioquia, Medellín.

Covián, O. N. (2005). El papel del conocimiento matemático en la construcción de la vivienda tradicional: el caso de la cultura Maya (Disertación de Maestría). Centro de Investigación y Estudios Avanzandos del Instituto Politécnico Nacional-CINVESTAV, México.

Chilisa, B.(2012). Indigenous research methodologies. Los Angeles, London, New Delhi, Singapore, Washintog DC: SEGA.

D’Ambrosio, U. (2008) Etnomatemática. Entre las tradiciones y la modernidad. México: Limusa.

D'Ambrosio, U. (2011). A busca da paz como responsabilidade dos matemáticos. Cuadernos de investigación y formación en educación matemática, (7). Recuperado de http://www. cimm.ucr.ac.cr/ojs/index.php/CIFEM/article/ view/664/653

Higuita, C. (2011). La medida desde la medicina tradicional: el caso de una comunidad Embera Chamí (Trabajo Grado). Universidad de Antioquia, Medellín.

Jaramillo, D. Tamayo, C. \& Higuita, C. (2013). Prácticas sociales, currículo y conocimiento matemático. (Informe).
Joutard, P. (1999). Esas voces que nos llegan del pasado. México, Argentina, Brasil, Colombia, Chile, España, Estados Unidos de América, Perú, Venezuela: Fondo de Cultura Académica

Lanner, A., Miguel, A. \& Vilela, D. (2012). [Problematização indisciplinar de uma prática cultural numa perspectiva wittgensteiniana]. Datos no publicados.

Larrosa, J. (2001). Dar la palabra. Notas para una dialógica de la transmisión. En J. Larrosa \& C. Skliar. (Eds.). Habitantes de babel: políticas y poéticas de la diferencia (pp. 411-431). España: Laertes.

Mendes, J. (2001). Ler, escrever e contar: práticas de numeramento-letramento dos Kaiabi no contexto de formação de professores índios no Parque Indígena do Xingu (Tesis de Doctorado). Campinas, Universidad Estadual de Campinas.

Miguel, A. (2008). Jogos hedonistas de lenguagem. São Paulo: Editora Plêiade.

Miguel, A. (2010, abril). Percursos Indisciplinares na Atividade de Pesquisa em História (da Educação Matemática): entre jogos discursivos como práticas e práticas como jogos discursivos. Bolema. 23 (35A), 1-57.

Sánchez, G. (1998). Fundamentos para la investigación educativa. Santa Fe de Bogotá: Cooperativa Editorial Magisterio.

Schaefer, C. (2008). La voz de las trece abuelas: ancianas indígenas aconsejan al mundo. Barcelona: Luciérnaga.

Tamayo, C. (2012). Resignificación del currículo escolar indígena, relativo al conocimiento [matemático], desde y para las prácticas sociales: el caso de lso maestros indígenas dule de la comunidad de Alto Caiman (Disertación de Maestría). Universidad de Antioquia, Medellín.

Walsh, C. (2005, enero-junio). Interculturalidad, conocimientos y decolonialidad. Signo y pensamiento 46 (24), 39-50.

Walsh, C. (2007, mayo/agosto). Interculturalidad, colonialidad y educación. Revista Educación y Pedagogía. 19 (48), 25-35. 